\title{
AFFINE CONNECTIONS AND DEFINING FUNCTIONS OF REAL HYPERSURFACES IN $\mathrm{C}^{n}$ \\ BY \\ HING-SUN LUK
}

\begin{abstract}
The affine connection and curvature introduced by Tanaka on a strongly pseudoconvex real hypersurface are computed explicitly in terms of its defining function. If Fefferman's defining function is used, then the Ricci form is shown to be a function multiple of the Levi form. The factor is computable by Fefferman's algorithm and its positivity implies the vanishing of certain cohomology groups (of the $\bar{\partial}_{b}$ complex) in the compact case.
\end{abstract}

Introduction. Let $M$ be a strongly pseudoconvex real hypersurface in $\mathbf{C}^{n}$. Let $\theta$ be a nowhere vanishing real differential form on $M$ which annihilates the complex tangent space at each point. It is well known that there exists a unique vector field $\xi$ on $M$ such that $\theta(\xi)=1$ and $\xi\lrcorner d \theta=0$. In [5], Tanaka has constructed a unique affine connection $\nabla$ satisfying certain natural conditions with respect to $\xi$. Using this canonical affine connection associated to $\xi$ (or $\theta$ ), he obtained the following Bochner-Kodaira type formula for the boundary Laplacian $\square_{b}$ (see $[5$, p. 47] and compare [4, p. 119]). Let $T^{\prime} M$ be the bundle of $(1,0)$ vectors tangent to $M$. Then

$$
\begin{array}{r}
\square_{b} \phi=-\frac{n-q-1}{n-1} \sum_{\alpha=1}^{n-1} \nabla_{x_{\alpha}} \nabla_{\bar{x}_{\alpha}} \phi-\frac{q}{n-1} \\
\sum_{\alpha=1}^{n-1} \nabla_{\bar{x}_{\alpha}} \nabla_{x_{\alpha}} \phi+\frac{n-q-1}{n-1} R_{*}(\phi),
\end{array}
$$

where $\phi$ is a smooth section of $\Lambda^{q}\left(\overline{T^{\prime} M}\right)^{*},\left\{X_{\alpha}\right\}_{\alpha-1, \ldots, n-1}$ is a local orthonormal basis of $T^{\prime} M$ with respect to the Levi metric defined by $\theta$ and $R_{*}$ is the Ricci operator associated to $\nabla$. In case $M$ is compact, (*) implies, by standard arguments, that if the selfadjoint operator $R_{*}$ is positive definite everywhere, then there is no nonzero harmonic form $\phi$ on $M$ and the cohomology groups $H^{0, q}(M)$ of the $\bar{\partial}_{b}$ complex vanish, for $q \neq 0, n-1 . H^{0, q}(M)$ here coincides with $H^{0, q}(\mathscr{B})$ in KohnRossi [3, p. 466]. In passing, the above theory works for an abstractly given strongly pseudoconvex CR manifold, not necessarily imbedded as a real hypersurface in $\mathbf{C}^{n}$.

There is a choice of $\xi($ or $\theta)$ in the construction of $\nabla$. One naturally asks if there exists a most natural choice. For any defining function $f$ on $M$, one may take $\theta=-i \partial f$. Now Fefferman [1] has constructed special defining functions which

Received by the editors February 13, 1979 and, in revised form, September 4, 1979.

AMS (MOS) subject classifications (1970). Primary 53C05, 53B05; Secondary 32F15, 35N15.

Key words and phrases. Strongly pseudoconvex real hypersurface, affine connection, Ricci operator, defining function, Levi form, boundary Laplacian. 
satisfy a Monge-Ampere equation to order $\leqslant n+1$ at $M$. The Monge-Ampere equation arises from considerations of the Bergman kernel, and is intimately related to the geometry of $M$. In this paper, we will show that if $f$ satisfies the equation to second order at $M$, then for the affine connection associated to $\boldsymbol{\theta}=-i \partial f$, the Ricci form is a function multiple of the Levi form. Thus, we obtain a real-valued function $\lambda$ on $M$, computable using Fefferman's algorithm, such that the Ricci operator is positive definite at a point $x$ in $M$ if and only if $\lambda(x)>0$. If $\lambda$ is everywhere positive, then the cohomology groups $H^{0, q}(M)(q \neq 0, n-1)$ vanish. Relevant to an open problem posed in [1] concerning intrinsic development, it would be interesting to know if there is some natural choice of $\theta$ for constructing $\nabla$ on a CR manifold.

In $\S 1$, we state explicitly all the results from [1] and [5] which we need. In §2, we consider any defining function $f$ and derive formulas for the affine connection and curvature associated to $\theta=-i \partial f$. It is interesting to compare our formulas to the local formulas of Riemannian and Kähler geometry as in [2]. We apply the formulas to consider the ellipsoids and to prove the main theorem in the last section. The formulas apply also to real hypersurfaces in complex manifolds; we shall return to their applications to isolated singularities (cf. [5]) later.

I would like to thank Professors Masatake Kuranishi and Shiu-Yuen Cheng for their encouragement and helpful discussions.

1. Notations and preliminaries. Throughout this paper, we use the notation of tensor calculus. Small Greek (resp. Latin) indices always run from 1 to $n-1$ (resp. 1 to $n$ ). Summation over repeated indices is understood. $z^{1}, \ldots, z^{n}$ are the coordinates of $\mathbf{C}^{n}$, and $f_{j}, f_{\bar{k}}, f_{j \bar{k}}, \ldots$ stand for the partial derivatives $\partial f / \partial z^{j}, \partial f / \partial \overline{z^{k}}, \partial^{2} f / \partial z^{j} \partial \overline{z^{k}}, \ldots$. For a $C^{\infty}$ manifold $M, T M, T^{*} M$, etc. have the usual meaning. For a vector bundle $V$ over $M, C V=V \otimes C, \Gamma(V)$ denotes the space of $C^{\infty}$ sections in $V$, and $V_{x}$ denotes the fiber over $x \in M$.

Let $M$ be a $C^{\infty}$ strongly pseudoconvex real hypersurface in $C^{n}$. Let $T^{\prime} M$ (resp. $\left.T^{\prime \prime} M\right)$ be the subbundle of $C T M$ consisting of vectors of type $(1,0)($ resp. $(0,1))$ in $\mathrm{C}^{n}$. There is a subbundle $H(M)$ of $T M$ and a homomorphism $I: H(M) \rightarrow H(M)$ such that

(1) $\mathrm{CH}(M)=T^{\prime} M \oplus T^{\prime \prime} M$.

(2) $I^{2}=-1$ and $T^{\prime} M=\{X-i I X \mid X \in H(M)\}$.

At each point $x \in M, H_{x}(M)$ is called the complex tangent space at $x$. Let $\theta \in \Gamma\left(T^{*} M\right)$ be any nowhere vanishing differential form on $M$ which annihilates $H(M)$. There is a unique vector field $\xi$ on $M$ such that

$$
\theta(\xi)=1 \text { and } \xi\lrcorner d \theta=0 .
$$

Extend $I$ to a tensor field of type $(1,1)$ on $M$ by setting $I(\xi)=0$. Then,

$$
I^{2}(X)=-X+\theta(X) \xi \text { for any } X \in T M .
$$

Let $\omega=-d \theta$. We shall consider the Levi form $\langle$,$\rangle defined by$

$$
\langle X, Y\rangle=\omega(I X, Y), \quad X, Y \in \mathbf{C} T_{x} M, \quad x \in M .
$$


Further we assume that $\langle$,$\rangle is positive definite on H(M)$. Let $E$ be the 1-dimensional subbundle of $\mathbf{C} T M$ spanned by $\xi$. Thus, for each $x \in M$, the fiber $E_{x}=\mathbf{C} \xi_{x}$. For any $X \in C T M$, we denote by $X_{T^{\prime}}$ (resp. $\left.X_{T^{\prime \prime}}, X_{E}\right)$ the $T^{\prime} M$ - (resp. $T^{\prime \prime} M-, E-$ ) component of $X$ with respect to the decomposition $C T M=T^{\prime} M \oplus T^{\prime \prime} M \oplus E$.

Now we recall Tanaka's canonical affine connection on $M$ associated to $\xi$. This is a connection

$$
\nabla: \Gamma(T M) \rightarrow \Gamma\left(T M \otimes T^{*} M\right)
$$

on $M$ satisfying the following conditions:

(5) $\nabla_{X} \Gamma(H(M)) \subset \Gamma(H(M))$ for any $X \in \Gamma(T M)$ i.e. $H(M)$ is parallel.

(6) The tensor fields $\xi, I, \omega$ are parallel.

(7) Let $T$ be the torsion of $\nabla$. For any $X, Y \in \Gamma(H(M))$,

$$
T(X, Y)=-\omega(X, Y) \xi \text { and } T(\xi, I Y)=-I T(\xi, Y) .
$$

We shall use the following formulas [5, p. 31]. Let $X, Y \in \Gamma\left(T^{\prime} M\right)$.

(8) $\nabla_{\bar{X}} Y=[\bar{X}, Y]_{T^{\prime}}$

(9) $\nabla_{X} Y \in \Gamma\left(T^{\prime} M\right)$ and $\left\langle\nabla_{X} Y, \bar{W}\right\rangle=X\langle Y, \bar{W}\rangle-\left\langle Y, \overline{\nabla_{\bar{X}} W}\right\rangle$ for all $W \in$ $\Gamma\left(T^{\prime} M\right)$.

(10) $\nabla_{\xi} Y=L_{\xi} Y-\frac{1}{2} I\left(L_{\xi} I\right) Y$, where $L_{\xi}$ denotes the Lie derivation.

Finally, we recall Fefferman's defining functions. Let

$$
J(u)=(-1)^{n} \operatorname{det}\left(\begin{array}{cccc}
u & u_{\overline{1}} & \cdots & u_{\bar{n}} \\
u_{1} & u_{1 \overline{1}} & \cdots & u_{1 \bar{n}} \\
\vdots & \vdots & & \vdots \\
u_{n} & u_{n \overline{1}} & \cdots & u_{n \bar{n}}
\end{array}\right),
$$

where $u$ is any smooth function. In [1], Fefferman considered defining equations of $M$ satisfying

$(11)^{\prime} J(u)=1$, to high order at $M$.

He also found a clever algorithm for $u$ up to order $n+1$.

2. Formulas for connection and curvature. Let $f$ be any defining function of $M$. Precisely, let $f$ be a $C^{\infty}$ real-valued function defined on some neighborhood of $M$ such that $M$ is defined by the equation $f=0$, and $d f \neq 0$ on $M$. Take $\theta=-i \partial f$. The assumption on $\langle$,$\rangle implies that f_{j \bar{k}} w^{j} \overline{w^{k}}>0$ for any nonzero vector $w^{j} \partial / \partial z^{j}$ satisfying $w^{j} f_{j}=0$. The matrix $\left(f_{j \bar{k}}\right)$ need not be invertible. Let $\xi=\xi^{j} \partial / \partial z^{j}$ $+\overline{\xi^{j}} \partial / \partial \bar{z}^{j}$. Condition (3) is equivalent to

$$
i f_{\bar{k}} \overline{\xi^{k}}=1
$$

and

$$
x^{j} f_{j}=0 \quad \text { implies } x^{j} f_{j \bar{k}} \overline{\xi^{k}}=0 .
$$

Choose a local $C^{\infty}$ orthonormal basis $\left\{X_{\alpha}=x_{\alpha}^{j} \partial / \partial z^{j}\right\}_{\alpha=1, \ldots, n-1}$ of $T^{\prime} M$ with respect to $\langle$,$\rangle . Thus,$

$$
x_{\alpha}^{j} f_{j}=0
$$


and

$$
x_{\alpha}^{j} f_{j \bar{k}} \overline{x_{\beta}^{k}}=\delta_{\alpha}^{\beta}
$$

Proposition 1. Let

and

$$
F=\left(\begin{array}{cccc}
f & f_{\overline{1}} & \cdots & f_{\bar{n}} \\
f_{1} & f_{1 \overline{1}} & \cdots & f_{1 \bar{n}} \\
\vdots & \vdots & & \vdots \\
f_{n} & f_{n \overline{1}} & \cdots & f_{n \bar{n}}
\end{array}\right)
$$

$$
A=\left(\begin{array}{cccc}
-\langle\xi, \xi\rangle^{\prime} & -i \xi^{1} & \ldots & -i \xi^{n} \\
i \overline{\xi^{1}} & a^{\overline{1} 1} & \cdots & a^{\overline{1} n} \\
\vdots & \vdots & & \vdots \\
i \overline{\xi^{n}} & a^{\bar{n} 1} & \cdots & a^{\bar{n} n}
\end{array}\right),
$$

where $a^{j k}=\overline{x_{\alpha}^{j}} x_{\alpha}^{k}$ and $\langle\xi, \xi\rangle^{\prime}=\frac{1}{2}\langle\xi, \xi\rangle$. Then $F A=I_{n+1}$.

Proof. $f_{j} d z^{j}$ and $f_{j k} \bar{\xi}^{k} d z^{j}$ annihilate all $X_{\alpha}$, hence they are linearly dependent. Since $d f \neq 0$, we may assume that $f_{j k} \bar{\xi}^{k}=a f_{j}$. Contracting with $\xi^{j}$ gives $a=$ $-i\langle\xi, \xi\rangle^{\prime}$. Thus,

$$
-f_{j}\langle\xi, \xi\rangle^{\prime}+i f_{j \bar{k}} \overline{\xi^{k}}=0
$$

Writing $\xi^{j}=x_{n}^{j}$, we have by (3) $)_{2}$ and (13)

$$
\begin{aligned}
& \left(\begin{array}{ccc}
x_{1}^{1} & \cdots & x_{1}^{n} \\
\vdots & & \vdots \\
x_{n}^{1} & \cdots & x_{n}^{n}
\end{array}\right]\left(\begin{array}{ccc}
f_{1 \overline{1}} & \cdots & f_{1 \bar{n}} \\
\vdots & & \vdots \\
f_{n \overline{1}} & \cdots & f_{n \bar{n}}
\end{array}\right]\left[\begin{array}{ccc}
\overline{x_{1}^{1}} & \cdots & \overline{x_{n}^{1}} \\
\vdots & & \vdots \\
\overline{x_{1}^{n}} & \cdots & \overline{x_{n}^{n}}
\end{array}\right)
\end{aligned}
$$

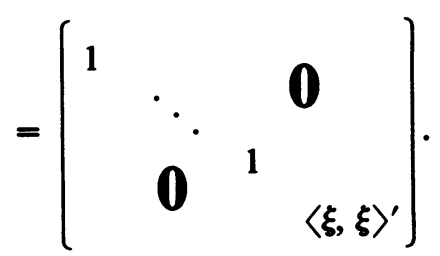

$\left(x_{j}^{k}\right)$ is invertible because $\xi^{j} \partial / \partial z^{j} \notin T^{\prime} M$. Let $\left(y_{j}^{k}\right)=\left(x_{j}^{k}\right)^{-1}$, noting that $y_{j}^{n}=-i f_{j}$ by (3) 1 and (12). Then

$$
\left(f_{j i}\right)\left(\overline{x_{r}^{l}}\right)\left(x_{r}^{k}\right)=\left(y_{j}^{l}\right)\left[\begin{array}{cccc}
1 & & & \\
& \ddots & & 0 \\
& 0 & 1 & \\
& & & \langle\xi, \xi\rangle^{\prime}
\end{array}\right]\left(x_{r}^{k}\right) .
$$


Now

$$
f_{j i} \overline{x_{r}^{l}} x_{r}^{k}=f_{j i l}\left(\overline{x_{\alpha}^{l}} x_{\alpha}^{k}+\overline{\xi^{l}} \xi^{k}\right)=f_{j i} a^{\overline{l k}}-i\langle\xi, \xi\rangle^{\prime} f_{j} \xi^{k} \quad \text { by (14), }
$$

while the $(j, k)$-element on the right of $(15)^{\prime}$ is

$$
y_{j}^{\alpha} x_{\alpha}^{k}+\langle\xi, \xi\rangle^{\prime} y_{j}^{n} x_{n}^{k}=\delta_{j}^{k}+\left(\langle\xi, \xi\rangle^{\prime}-1\right) y_{j}^{n} x_{n}^{k}=\delta_{j}^{k}+i f_{j} \xi^{k}-i\langle\xi, \xi\rangle^{\prime} f_{j} \xi^{k} \text {. }
$$

Therefore, we get

$$
-i f_{j} \xi^{k}+f_{j l} a^{\overline{l k}}=\delta_{j}^{k}
$$

By (12),

$$
f_{j} a^{\overline{j k}}=0 .
$$

Since $f=0$ on $M,(3)_{1},(14),(16),(17)$ prove the proposition.

We shall use the following notations:

$$
\begin{aligned}
& F=\left(\begin{array}{ccc}
f_{\overline{0} \overline{0}} & \cdots & f_{0 \bar{n}} \\
\vdots & & \vdots \\
f_{n \overline{0}} & \cdots & f_{n \bar{n}}
\end{array}\right), \\
& A=\left(\begin{array}{ccc}
a^{\overline{0} 0} & \cdots & a^{\overline{0} n} \\
\vdots & & \vdots \\
a^{\bar{n} 0} & \cdots & a^{\bar{n} n}
\end{array}\right) .
\end{aligned}
$$

Proposition 1 implies that $F$ is invertible in a neighborhood of $M$. Let $A=F^{-1}$ and use (18) to extend the functions $a^{\bar{k} l}, a^{\overline{k 0}}=i \xi^{\bar{k}}$ and $a^{\overline{0} 0}=a$. Then in a neighborhood of $M$, we have

$$
\begin{gathered}
a f+i f_{\bar{k}} \overline{\xi^{k}}=1, \\
a f_{j}+i f_{j \bar{k}} \overline{\xi^{k}}=0, \\
-i f_{j} \xi^{l}+f_{j \bar{k}} a^{\overline{k l}}=\delta_{j}^{l}, \\
-i f \xi^{l}+f_{\bar{k}} a^{\bar{k} l}=0 .
\end{gathered}
$$

Note that $\xi^{j} \partial / \partial z^{j}+\overline{\xi^{j}} \partial / \partial \bar{z}^{j}$ is not the vector field corresponding to $\theta=-i \partial f$ on each level real hypersurface unless $a=0$.

We now give local formulas corresponding to (8), (9) and (10).

PROPOSITION 2. Let $X=x^{j} \partial / \partial z^{j}$ and $Y=y^{j} \partial / \partial z^{j}\left(\right.$ with $\left.x^{j} f_{j}=y^{j} f_{j}=0\right)$ be local $C^{\infty}$ sections in $T^{\prime} M$. Then

(a) $\nabla_{\bar{X}} Y=\left(\bar{X}\left(y^{k}\right)+\bar{x}^{j} y^{l} \Gamma_{j l}^{k}\right) \partial / \partial z^{k}$, where $\Gamma_{j l}^{k}=-i f_{j l} \xi^{k}$.

(b) $\nabla_{X} Y=\left(X\left(y^{k}\right)+x^{j} y^{l} \Gamma_{j l}^{k}\right) \partial / \partial z^{k}$,

where $\Gamma_{j l}^{k}=\sum_{A=0}^{n} a^{\bar{A} k}\left(\partial / \partial z^{j}\right) f_{L \bar{A}}=\Gamma_{l j}^{k}$.

(c) $\nabla_{\xi} Y=\left\{\xi\left(y^{k}\right)-Y\left(\xi^{k}\right)\right\} \partial / \partial z^{k}$.

Proof. We first derive

$$
\nabla_{\xi} Y=[\xi, Y]_{T^{\prime}}
$$


Recall $\theta(Y)=0$ and $I Y=i Y$. Using (3), note that $\theta([\xi, Y])=0$. Then

$$
\begin{aligned}
\nabla_{\xi} Y & =[\xi, Y]+\frac{1}{2} I(I[\xi, Y]-[\xi, I Y]) \text { by }(10) \\
& =[\xi, Y]-\frac{1}{2}[\xi, Y]-\frac{i}{2} I[\xi, Y] \text { by }(4) \\
& =\frac{1}{2}\left([\xi, Y]_{T^{\prime}}+[\xi, Y]_{T^{\prime \prime}}\right)-\frac{i}{2}\left(i[\xi, Y]_{T^{\prime}}-i[\xi, Y]_{T^{\prime \prime}}\right)=[\xi, Y]_{T^{\prime}}
\end{aligned}
$$

Next note that for any $Z=a^{j} \partial / \partial z^{j}+b^{j} \partial / \partial \overline{z^{j}}$ in CTM,

$$
\begin{aligned}
Z_{E} & =\theta(Z) \xi, \quad \theta(Z)=-i a^{l} f_{l}=i b^{\bar{l}} f_{\bar{l}} . \\
Z_{T^{\prime}} & =\left(a^{k}+i a^{l} f_{l} \xi^{k}\right) \frac{\partial}{\partial z^{k}} . \\
Z_{T^{\prime \prime}} & =\left(b^{\bar{k}}-i b^{\bar{l}} \bar{f}_{\bar{l}} \overline{\xi^{k}}\right) \frac{\partial}{\partial \overline{z^{k}}} .
\end{aligned}
$$

(a) and (c) then follow easily from (8) and (10)', using $\theta([\bar{X}, Y])=i\langle Y, \bar{X}\rangle$ and $\theta([\xi, Y])=0$.

To get (b), observe that for any $W=w^{i} \partial / \partial z^{j}$ with $w^{j} f_{j}=0$,

$$
\begin{aligned}
X\langle Y, \bar{W}\rangle-\left\langle Y, \overline{\nabla_{\bar{X}} W}\right\rangle & =X\left(y^{k}\right) f_{k \bar{m}} \overline{w^{m}}+x^{j} y f_{j l \bar{m}} \overline{w^{m}} \text { since } y^{j} f_{j \bar{k}} \overline{\Gamma_{l m}^{k}}=0 \\
& =\left(X\left(y^{k}\right)+x^{j} y f_{j l i} a^{i k}\right) f_{k \bar{m}} \overline{w^{m}} \text { by (16) } \\
& =\left\{X\left(y^{k}\right)+x^{j} y^{l}\left(a^{i k_{j l i}}-i f_{j l} \xi^{k}\right)\right\} f_{k \bar{m}} \overline{w^{m}} \text { by (3) }{ }_{2} .
\end{aligned}
$$

Let $v^{k}=X\left(y^{k}\right)+x^{j} y^{l}\left(a^{i k} f_{j l i}-i f_{j l} \xi^{k}\right)$. By (3), and (17), $v^{k} f_{k}=0$, hence $v^{k} \partial / \partial z^{k}$ $\in \Gamma\left(T^{\prime} M\right)$. By (9), $\nabla_{X} Y=v^{k} \partial / \partial z^{k}$. The expression for $\Gamma_{j l}^{k}$ simply follows from notations in (18) and (18)', finishing the proof.

In the following, we consider $\Gamma_{j k}^{l}$ and $\Gamma_{j k}^{l}$ as functions on a neighborhood of $M$, defined by the formulas in Proposition 2.

Proposition 3. Let $X=x^{j} \partial / \partial z^{j}, Y=y^{j} \partial / \partial z^{j}$ and $W=w^{j} \partial / \partial z^{j}$ be $C^{\infty}$ sections in $T^{\prime} M$. Then

$$
R(X, \bar{Y}) W=\left(\nabla_{X} \nabla_{\bar{Y}}-\nabla_{\bar{Y}} \nabla_{X}-\nabla_{[X, \bar{Y}]}\right) W=x^{j} \overline{y^{k}} w^{l} R_{j k l}^{p} \frac{\partial}{\partial z^{p}},
$$

where

$$
R_{j k l}^{p}=\frac{\partial}{\partial z^{j}} \Gamma_{k l}^{p}+\frac{\partial}{\partial z^{l}} \Gamma_{k j}^{p}-\frac{\partial}{\partial \overline{z^{k}}} \Gamma_{j l}^{p}+\Gamma_{\overline{k l}}^{r} \Gamma_{j r}^{p}+\Gamma_{k j}^{r} \Gamma_{r l}^{p}-\Gamma_{j l}^{r} \Gamma_{k r}^{p}+i f_{j \overline{k l}} \xi^{p}
$$

and

$$
R_{j k l}^{p} f_{p} \equiv 0 \quad \bmod f_{j}, f_{\bar{k}}, f_{l}
$$

Proof. Decomposing $[X, \bar{Y}]$ by (19) a,b,c and using Proposition 2, one obtains by straightforward computation

$$
\begin{array}{r}
R(X, \bar{Y}) W=\left\{x^{j} \bar{y}^{\bar{k}} w^{l}\left(\frac{\partial}{\partial z^{j}} \Gamma_{k l}^{p}-\frac{\partial}{\partial \overline{z^{k}}} \Gamma_{j l}^{p}+\Gamma_{\overline{k l}}^{r} \Gamma_{j r}^{p}-\Gamma_{j l}^{r} \Gamma_{k r}^{p}\right)\right. \\
\left.-i\langle X, \bar{Y}\rangle\left(\xi^{r} w^{l} \Gamma_{r l}^{p}+\overline{\xi^{r}} w^{l} \Gamma_{\bar{l} l}^{p}+W\left(\xi^{p}\right)\right)\right\} \frac{\partial}{\partial z^{p}}
\end{array}
$$


REAL HYPERSURFACES IN C

585

Now

$$
-i\langle X, \bar{Y}\rangle \xi^{r} w^{l} \Gamma_{r l}^{p}=x^{j} \overline{y^{k}} w^{l} \Gamma_{\overline{k j}}^{r} \Gamma_{r l}^{p}, \quad \overline{\xi^{r}} \Gamma_{\overline{r l}} w^{l}=0
$$

and

$$
-i\langle X, \bar{Y}\rangle W\left(\xi^{p}\right)=x^{j} \overline{y^{k}}\left(-i f_{j \bar{k}}\right) W\left(\xi^{p}\right)=x^{j} y^{\bar{k}} w^{l}\left(\frac{\partial}{\partial z^{l}} \Gamma_{k j}^{p}+i f_{j \bar{k}} \xi^{l}\right) .
$$

Hence

$$
R(X, \bar{Y}) W=x^{j} \overline{y^{k}} w^{l} R_{j k l}^{p} \frac{\partial}{\partial z^{p}}
$$

with $R_{j k l}^{p}$ as given.

Next note that $R(X, \bar{Y}) W \in \Gamma\left(T^{\prime} M\right)$ for any $X, Y, W \in \Gamma\left(T^{\prime} M\right)$. Hence $x^{j} y^{k} w^{l} R_{j k b}^{p} f_{p}=0$ whenever $x^{j} f_{j}=y^{j} f_{j}=w^{j} f_{j}=0$. The following lemma then implies that $R_{j k l}^{p} f_{p} \equiv 0 \bmod f_{j}, f_{\bar{k}}, f_{l}$, finishing the proof of the proposition.

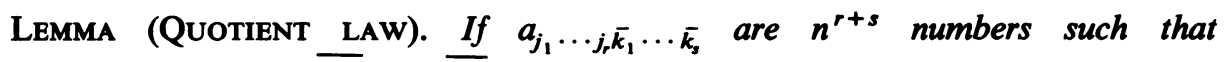
$a_{j_{1}} \cdots j_{r} \bar{k}_{1} \cdots \bar{k}_{s} x_{1}^{j_{1}} \cdots x_{r}^{j_{r}} \overline{y_{1}^{k_{1}}} \cdots \overline{y_{s}^{k_{s}}}=0$ whenever $x_{i}^{j_{j}} f_{j}=0(i=1, \ldots, r)$ and $y_{l}{ }^{k} f_{k}=$ $0(l=1, \ldots, s)$, then $a_{j_{1}} \cdots j_{r} \bar{k}_{1} \ldots \bar{k}_{s} \equiv 0 \bmod f_{j_{1}}, \ldots, f_{j_{j}}, f_{\bar{k}_{1}}, \ldots, f_{\bar{k}_{s}}$.

Proof. For simplicity we prove the case $r=s=1$; the general case is similar. Since $d f \neq 0$, we assume that $f_{n} \neq 0$. Consider

$$
x=\left(0, \ldots, \underset{(\alpha)}{1}, \ldots, 0,-f_{\alpha} / f_{n}\right) \text { and } y=\left(0, \ldots, \underset{(\beta)}{1}, \ldots, 0,-f_{\beta} / f_{n}\right)
$$

in $a_{j \bar{k}} x^{j} \overline{y^{k}}$. We get

$$
a_{\alpha \bar{\beta}}=\frac{f_{\alpha}}{f_{n}} a_{n \bar{\beta}}+\frac{f_{\bar{\beta}}}{f_{n}} a_{\alpha \bar{n}}-\frac{f_{\alpha} f_{\bar{\beta}}}{f_{n}^{2}} a_{n \bar{n}}
$$

and verify that

$$
a_{j \bar{k}}=\frac{f_{j}}{f_{n}} a_{n \bar{k}}+\frac{f_{\bar{k}}}{f_{n}} a_{j \bar{n}}-\frac{f_{j} f_{\bar{k}}}{f_{n}^{2}} a_{n \bar{n}}
$$

finishing the proof.

Proposition 4. Let $X=x^{j} \partial / \partial z^{j}, Y=y^{j} \partial / \partial z^{j}, W=w^{j} \partial / \partial z^{j}$ and $U=u^{j} \partial / \partial z^{j}$ be $C^{\infty}$ sections in $T^{\prime} M$. Then

$$
\langle R(X, \bar{Y}) W, \bar{U}\rangle=R_{j \bar{k} l \bar{m}} x^{j} \overline{y^{k}} w^{l} \overline{u^{m}},
$$

where

$$
\begin{aligned}
R_{j \bar{k} l m}= & -f_{j \bar{k} \bar{m}}+f_{j \bar{r}} a^{\bar{r} s} f_{s \bar{k} \bar{m}}-i\left(f_{j l} f_{\bar{k} \bar{m} r} \xi^{r}-f_{\bar{k} \bar{m}} f_{j l} \overline{\xi^{s}}\right) \\
& +\langle\xi, \xi\rangle^{\prime}\left(f_{j \bar{k}} f_{l \bar{m}}+f_{l \bar{k}} f_{j \bar{m}}-f_{j l} f_{\bar{k} \bar{m}}\right) .
\end{aligned}
$$


Proof. It suffices to compute $R_{j k l}^{p} f_{\bar{p} \bar{m}} \bmod f_{\bar{m}}$. There are seven terms:

$$
\begin{aligned}
& \left(\frac{\partial}{\partial z^{j}} \Gamma_{k l}^{p}\right) f_{p \bar{m}}=-\left(f_{j \overline{k l}} \xi^{p} f_{p \bar{m}}+f_{\overline{k l}} \frac{\partial \xi^{p}}{\partial z^{j}} f_{p \bar{m}}\right) \\
& \equiv\langle\xi, \xi\rangle^{\prime} f_{j \bar{m}} f_{\overline{l k}}+i f_{\bar{k}} f_{\bar{m} j p} \xi^{p} \text { by (14) and (14)'. } \\
& \left(\frac{\partial}{\partial z^{l}} \Gamma_{k j}^{p}\right) f_{p \bar{m}}=\langle\xi, \xi\rangle^{\prime} f_{l \bar{m}} f_{j \bar{k}}+i f_{\overline{k j}} f_{\bar{m} m p} \xi^{p} \text {. } \\
& -\left(\frac{\partial}{\partial \overline{z^{k}}} \Gamma_{j l}^{p}\right) f_{p \bar{m}}=-f_{j l i} \frac{\partial a^{\bar{p}}}{\partial \overline{z^{k}}} f_{p \bar{m}}-f_{j \overline{k l} l} a^{\bar{i}} f_{p \bar{m}}+i f_{j l} \frac{\partial \xi^{p}}{\partial \overline{z^{k}}} f_{p \bar{m}}+i f_{j l \bar{k}} \xi^{p} f_{p \bar{m}} \\
& \equiv f_{j l i} a^{\bar{p} p} f_{p \bar{m} \bar{k}}+i f_{j l i} f_{\bar{k} \bar{m}} \xi^{\bar{i}}-f_{j \bar{k} l \bar{m}} \\
& -\langle\xi, \xi\rangle^{\prime} f_{j l} f_{\bar{m} \bar{k}}-i f_{j l} f_{\bar{m} \bar{k} p} \xi^{p} \text { by (14), (14)', (16), (16)'. }
\end{aligned}
$$

By (14) and (16),

$$
\begin{gathered}
\Gamma_{\bar{k} l}^{r} \Gamma_{j r}^{p} f_{p \bar{m}} \equiv-i f_{\bar{k} l} f_{\bar{m} j p} \xi^{p} \\
\Gamma_{\bar{k} j}^{r} \Gamma_{r h}^{p} f_{p \bar{m}} \equiv-i f_{\overline{k j}} f_{\bar{m} l p} \xi^{p} \\
-\Gamma_{j l}^{r} \Gamma_{k r}^{p} f_{p \bar{m}} \equiv 0 \\
i f_{j \overline{k l} l} \xi^{p} f_{p \bar{m}} \equiv 0
\end{gathered}
$$

The proposition follows immediately.

Observe that we have

$$
\begin{aligned}
R_{j \bar{k} l \bar{m}}= & R_{j \bar{m} l \bar{k}}=R_{l \bar{k} j \bar{m}}=R_{l \bar{m} \bar{k},} \\
R_{j \bar{k} l \bar{m}} & =R_{k \bar{j} \bar{l} \bar{l}}
\end{aligned}
$$

corresponding to the properties $[5$, p. 34]

$$
\begin{aligned}
&\langle R(X, \bar{Y}) W, \bar{U}\rangle=\langle R(X, \bar{U}) W, \bar{Y}\rangle \\
&=\langle R(W, \bar{Y}) X, \bar{U}\rangle=\langle R(W, \bar{U}) X, \bar{Y}\rangle, \\
&\langle R(X, \bar{Y}) W, \bar{U}\rangle=\langle W, \overline{R(Y, \bar{X}) U}\rangle .
\end{aligned}
$$

REMARKs. (a) Formulas for $R(\xi, \bar{Y}) W$ etc. are complicated and will not be used. We shall however consider the following Ricci operator

$$
R_{*}(Y)=\sum_{\alpha=1}^{n-1} R\left(X_{\alpha}, \bar{X}_{\alpha}\right) Y, \quad Y \in \Gamma\left(T^{\prime} M\right) .
$$

(b) For the real hyperquadric defined by $z^{\alpha} \overline{z^{\alpha}}+i\left(z^{n}-\overline{z^{m}}\right) / 2=0, \xi=2\left(\partial / \partial z^{n}\right.$ $\left.+\partial / \partial \overline{z^{m}}\right)$ and $\langle\xi, \xi\rangle^{\prime}=0$, hence $R_{j \bar{k} l \bar{m}}=0$. This is the flat case.

\section{Applications of local formulas.}

(A) Ellipsoids. Consider the ellipsoid $E$ in $\mathbf{C}^{n}$ defined by the equation

$$
\begin{aligned}
f= & a_{\alpha \beta} z^{\alpha} z^{\beta}+\overline{a_{\alpha \beta}} \overline{z^{\alpha}} \overline{z^{\beta}}+b_{\alpha \bar{\beta}} z^{\alpha} \overline{z^{\beta}} \\
& +a z^{n} z^{n}+\bar{a} \overline{z^{n}} \overline{z^{n}}+b z^{n} \overline{z^{n}}-1=0
\end{aligned}
$$


where $a_{\alpha \beta}$ is symmetric, $b_{\alpha \bar{\beta}}\left(=\overline{b_{\beta \bar{\alpha}}}\right)$ is positive definite and $b$ is positive. Clearly

$$
R_{j \bar{k} l \bar{m}}=\langle\xi, \xi\rangle^{\prime}\left(f_{j k} f_{l \bar{m}}+f_{l \bar{k}} f_{j \bar{m}}-f_{j k} f_{\bar{k} \bar{m}}\right) \text {. }
$$

It is easy to solve for $\langle\xi, \xi\rangle^{\prime}$ from the equation

$$
\left[\begin{array}{ccc}
0 & f_{\bar{\beta}} & f_{\bar{n}} \\
f_{\alpha} & b_{\alpha \bar{\beta}} & 0 \\
f_{n} & 0 & b
\end{array}\right]\left(\begin{array}{c}
-\langle\xi, \xi\rangle^{\prime} \\
i \overline{\xi^{\beta}} \\
i \overline{\xi^{n}}
\end{array}\right)=\left(\begin{array}{l}
1 \\
0 \\
0
\end{array}\right)
$$

Setting $\left(c^{\bar{\alpha} \beta}\right)=\left(b_{\alpha \bar{\beta}}\right)^{-1}$, one obtains

$$
\langle\xi, \xi\rangle\left(c^{\bar{\beta} \alpha} f_{\bar{\beta}} f_{\alpha}+b^{-1}\left|f_{n}\right|^{2}\right)=1 .
$$

Hence $\langle\xi, \xi\rangle^{\prime}>0$. A simple computation then shows that for any local section $Y$ and any local orthonormal basis $\left\{X_{\alpha}\right\}$ of $T^{\prime} M$,

$$
\left\langle R_{*}(Y), \bar{Y}\right\rangle=\langle\xi, \xi\rangle^{\prime} \sum_{\alpha}\left(\left\|X_{\alpha}\right\|_{B}^{2}\|Y\|_{B}^{2}+\left|\left\langle X_{\alpha}, \bar{Y}\right\rangle_{B}\right|^{2}-\left|\left\langle X_{\alpha}, Y\right\rangle_{A}\right|^{2}\right),
$$

where $\langle Z, \bar{W}\rangle_{B}=b_{\beta \bar{\gamma}} z^{\beta} \overline{w^{\gamma}}+b z^{n} \overline{w^{n}},\|Z\|_{B}^{2}=\langle Z, \bar{Z}\rangle_{B}$ and $\langle Z, W\rangle_{A}=a_{\beta \gamma} z^{\beta} w^{\gamma}$ $+a z^{n} w^{n}$.

Applying Tanaka's results, we obtain

Proposition 5. If $\left|a_{\beta \gamma}\right|$ and $|a|$ are small such that the right-hand side of (23) is everywhere positive for all $Y$, then there is no nonzero harmonic scalar form on $M$ and the cohomology groups $H^{0, q}(E)(q \neq 0, n-1)$ of the $\overline{\partial_{b}}$ complex vanish.

The same method was applied to the sphere by Tanaka [5, p. 63]. Both cases are however very special examples of a result of Kohn and Rossi (obtained by a different method) which states that the same cohomology groups vanish for the boundary of any bounded strongly pseudoconvex domain in $\mathbf{C}^{n}[3, \mathrm{p}$. 467].

(B) We now consider special defining functions. Observe that for any defining function $f$ of $M$, the equality

$$
\frac{\partial}{\partial z^{j}} \log |\operatorname{det} F|=\sum_{B, C=0}^{n} a^{\bar{B} C} \frac{\partial}{\partial z^{j}} f_{C \bar{B}}
$$

holds in a neighborhood of $M$.

THEOREM. (a) If $f$ satisfies $J(f)=$ constant $+\mathcal{\theta}\left(f^{s}\right)$, then $\Gamma_{j l}^{l}=\theta\left(f^{s-1}\right)$.

(b) If $f$ satisfies $J(f)=$ constant $+\mathcal{O}\left(f^{3}\right)$, then

$$
\left\langle R_{*}(Y), \bar{Y}\right\rangle=-i \frac{\partial \xi^{l}}{\partial z^{l}}\langle Y, \bar{Y}\rangle \text { for any } Y \in \Gamma\left(T^{\prime} M\right) \text {. }
$$

Proof.

$$
\Gamma_{j l}^{l}=a^{\bar{A} l} \frac{\partial}{\partial z^{j}} f_{L \bar{A}}=\frac{\partial}{\partial z^{j}} \log |\operatorname{det} F|-a^{\overline{A 0}} \frac{\partial}{\partial z^{j}} f_{0 \bar{A}}
$$

by (24) and

$$
-a^{\overline{A 0}} \frac{\partial}{\partial z^{j}} f_{0 \bar{A}}=a f_{j}+i f_{j \bar{k}} \overline{\xi^{k}}=0
$$


by $(14)^{\prime}$. Hence

$$
\Gamma_{j l}^{l}=\frac{\partial}{\partial z^{j}} \log |\operatorname{det} F|=\frac{\partial}{\partial z^{j}} \log |J(f)|,
$$

and (a) follows. One easily verifies as in the proof of Proposition 4 that

$$
R_{j k l}^{p} f_{p} \equiv f_{j k} f_{l}\langle\xi, \xi\rangle^{\prime} \bmod f_{j}, f_{\bar{k}} .
$$

Setting $\tilde{R}_{j k l}^{p}=R_{j k l}^{p}+i f_{j k} f_{l}\langle\xi, \xi\rangle^{\prime} \xi^{p}$, one has

$$
R(X, \bar{Y}) W=x^{j} \overline{y^{k}} w^{l} \tilde{R}_{j k l}^{p} \frac{\partial}{\partial z^{p}},
$$

where

$$
\tilde{R}_{j k k}^{p} f_{p} \equiv 0 \bmod f_{j}, f_{\bar{k}}
$$

Now

$$
\begin{aligned}
\left\langle R_{*}(Y), \bar{Y}\right\rangle & =\sum_{\alpha}\left\langle R(Y, \bar{Y}) X_{\alpha}, \overline{X_{\alpha}}\right\rangle \text { by }(20)^{\prime} \\
& =y^{j} \overline{y^{k}} x_{\alpha}^{l} \tilde{R}_{j k b}^{p} f_{p \bar{m}} \overline{x_{\alpha}^{m}}=y^{j} \overline{y^{k}} \tilde{R}_{j k l}^{p} f_{p \bar{m}} a^{\bar{m} l} \\
& =y^{j} \overline{y^{k}} \tilde{R}_{j \overline{k l} l}^{l} \text { by }(16) \text { and }(25) \\
& =y^{j} \overline{y^{k}}\left(\frac{\partial \Gamma_{\overline{k l}}^{l}}{\partial z^{j}}-i f_{j \bar{k}} \frac{\partial \xi^{l}}{\partial z^{l}}-f_{j \bar{k}}\langle\xi, \xi\rangle^{\prime}\right)
\end{aligned}
$$

using (a) and Proposition 3. One finishes the proof by noting that $\partial \Gamma_{k l}^{l} / \partial z^{j} \equiv$ $\langle\xi, \xi\rangle^{\prime} f_{j \bar{k}} \bmod f_{\bar{k}}$ by $(14)^{\prime}$.

$-i \partial \xi^{l} / \partial z^{l}$ is the function $\lambda$ referred to in the introduction.

\section{REFERENCES}

1. C. Fefferman, The Monge-Ampere equation, the Bergman kernel, and geometry of pseudoconvex domains, Ann. of Math. (2) 103 (1976), 395-416.

2. S. Kobayashi and K. Nomizu, Foundations of differential geometry, Wiley, New York, Vol. I, 1963; Vol. II, 1969.

3. J. Kohn and H. Rossi, On the extension of holomorphic functions from the boundary of a complex manifold, Ann. of Math. (2) 81 (1965), 451-472.

4. J. Morrow and K. Kodaira, Complex manifolds, Holt, Rinehart and Winston, New York, 1971.

5. N. Tanaka, A differential geometric study on strongly pseudo-convex manifolds, Lectures in Math., No. 9, Department of Math., Kyoto University, Kinokuniya Book-Store Co. Ltd., Tokyo, 1975. MR 53 \#3361.

Department of Mathematics, Science Centre, The Chinese University of Hong Kong, Shatin, HONG KONG 